\title{
Understanding the Contributing Factors to Nighttime Crashes at Freeway Mainline Segments
}

\author{
Hongyun Chen, Kristiansson Fanny \\ Department of Civil Engineering, Embry-Riddle Aeronautical University, Daytona Beach, FL, USA \\ Email: Hongyun.chen@erau.edu
}

How to cite this paper: Chen, H.Y. and Fanny, K. (2019) Understanding the Contributing Factors to Nighttime Crashes at Freeway Mainline Segments. Journal of Transportation Technologies, 9, 450-461. https://doi.org/10.4236/jtts.2019.94028

Received: May 10, 2019

Accepted: September 24, 2019

Published: September 27, 2019

Copyright (C) 2019 by author(s) and Scientific Research Publishing Inc. This work is licensed under the Creative Commons Attribution International License (CC BY 4.0).

http://creativecommons.org/licenses/by/4.0/

\begin{abstract}
This study investigated the crash contributing factors to the injury outcomes and the characteristics of the night time crashes at freeway mainline segments. Multinomial logit model (MNL) was selected to estimate the explanatory variables at a 95\% confidence level. The six-year crash data (2005-2010) were obtained in the State of Florida, USA and five injury level outcomes, no injury, possible injury, non-incapacitating injury, capacitating injury, and fatal injury, were considered. The no injury level was selected as the baseline category.
\end{abstract}

Keywords

Contributing Factors, Nighttime Crash, Injury Severity, Freeway Mainline

\section{Introduction}

Driving during nighttime tends to be riskier than day time [1]. Though vehicles travelling at night is about 25 percent of the total traffic, the nighttime crash rate remains high in the past decades, in general approximately 1.6 times of the daytime rate [2]. Additionally, the fatality rate of nighttime crash is much higher than the day time's rate. In 2011, 13,861 fatal crashes were occurred nighttime, which accounted for almost half of the fatal crashes in the US. Even though the fatality rate decreases by 1.8 percent from 2010 to 2011, the fatal crashes increased by 0.5 percent.

Figure 1 compares the five injury outcomes, no injury, possible injury, non-incapacitating injury, incapacitating injury, and fatal injury, in the State of Florida, USA from the year 2005 to 2010. Not only the fatal injury, but the also the percentages of incapacitating injury, and non-incapacitating injury are higher 


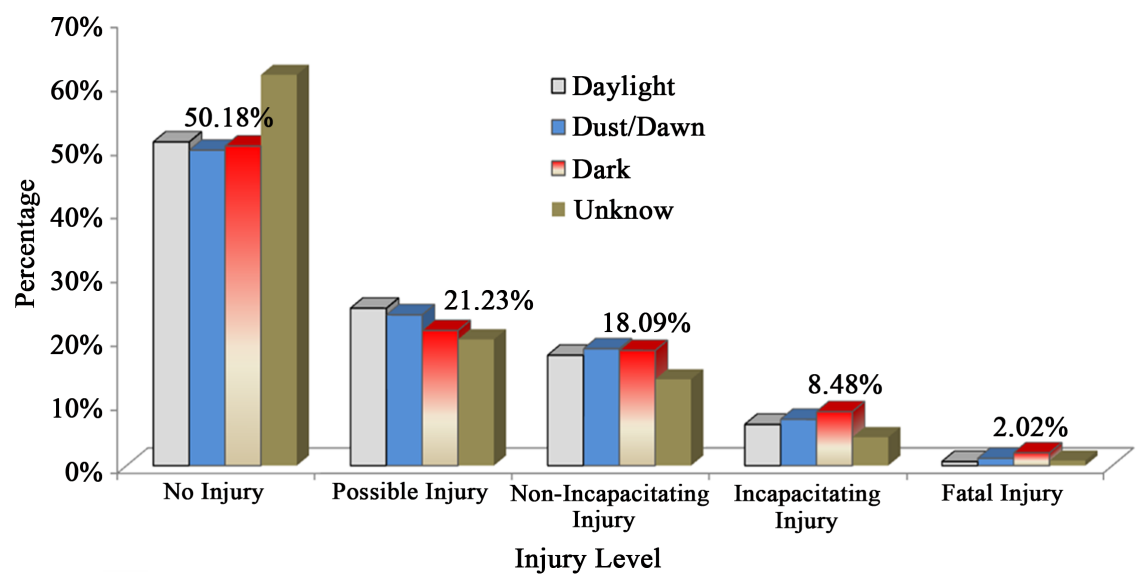

Figure 1. Percentage of crash injury levels under different lighting conditions from 2005-2010.

at the night time than those during the day time and dust/dawn conditions. The facts indicate there is a need to better understand the contributing factors to those nigh time crashes, and crash characteristics.

Few studies have focused on the night time crashes and most of them concentrated on demonstrating the benefit of using street lights to reduce night time crashes. Hawkins et al. [2] modeled the crashes with the presence of lighting and absence of lighting at 233 rural unsignalized intersections and found the locations without lighting had twice as many as crashes as locations with lighting. Elvik [3] compared the results from 37 studies of safety effects of public lighting and found that lightning reduces night time fatal accidents by 65 percent while 15 percent reduction for property damage only (PDO) crashes. However, a study conducted by Griffith [4] compared the night time crashes on a 55-mile long urban freeway segment with continuous lighting and a 36 mile without lighting. It only found the significant difference for PDO crash rates, which is 19 percent higher at those without street lighting.

These studies were conducted about 15 to 20 years ago while the sample sizes were limited, and detailed levels of crash information might not be available at that time which could significantly lead to the bias in the estimation.

Nighttime crashes appear more complicated than the day time crashes. The purpose of this study is to investigate the crash contributing factors to the injury outcomes and the characteristics of the night time crashes at freeway mainline segments. The findings from this study could help the engineers and researchers to further select the effective countermeasures or policies to effectively reduce the night time crashes, especially the injury severity levels.

\section{Methology}

Many statistical models have been applied to model injury severity levels. Multinomial logit model (MNL) and ordered probit model are the two commonly used ones [5] [6] [7] [8]. The benefit of using MNL is that each injury outcome has an individual function while the ordered model has the same coefficient of 
the same variable for the injury outcomes. It is likely that the ordered model might overestimate the probability of the high injury level while underestimates the low injury level [7]. Moreover, the same variable might significantly impact one injury level and not the other one. MNL is more flexible and suitable in this study. Each injury MNL could have different variables and coefficients which can distinguish the efforts of same variables on the different injury levels. Thus, the MNL in this study estimated the crash injury outcomes by different explanatory variables.

Assuming there are $m$ possible severity injury outcomes $(m=5$, no injury, possible injury, non-incapacitating injury, incapacitating injury, and fatal injury), the MNL model has a function for each injury outcome in the following equation [5] [9]:

$$
\boldsymbol{Y}_{i k}=\alpha_{k}+\boldsymbol{\beta}_{k} \boldsymbol{X}_{i k}+\varepsilon_{i k}, i=1, \cdots, n ; k=1, \cdots, m
$$

where $n$ is the number of total crashes; $\boldsymbol{Y}_{i k}$ is the severity function for the $k$ th possible injury outcome of the ith crash; $\alpha_{k}$ is an intercept parameter for the severity injury outcome $k, \boldsymbol{X}_{i k}$ is a vector of explanatory variables of crash $i$ in the injury outcome $k, \beta_{k}$ is a vector of coefficients to be estimated; and $\varepsilon_{i k}$ is an random error term following the Type I [10] distribution. The no injury outcome was used as the baseline category. Let $P_{i}(k)$ represent the probability of the crash $i$ in the severity injury outcome $k$, then the probability is:

$$
P_{i}(k \mid \boldsymbol{\beta})=\frac{\exp \left(\alpha_{k}+\boldsymbol{\beta}_{k} \boldsymbol{X}_{i k}+\varepsilon_{i k}\right)}{\sum_{k=1}^{m} \exp \left(\alpha_{m}+\boldsymbol{\beta}_{m} \boldsymbol{X}_{i m}+\varepsilon_{i m}\right)}
$$

The following equation determines the odds ratio (OR) or relative risk by using the estimated coefficients.

$$
\mathrm{OR}_{j_{k}}=\exp \left(\beta_{j_{k}}\right), \quad j_{k}=1, \cdots, u_{k}
$$

where $j_{k}$ is the explanatory variable in the injury outcome $k$ model and $u_{k}$ is the total number of significant explanatory variables which vary in different injury outcome models. The OR indicates the probability of choosing one outcome category over the probability of choosing the baseline category (no injury outcome) for one specific explanatory variable while others hold constant. If OR is larger than 1 , it is likely to increase the injury level outcome $k$, if OR is smaller than 1 , the injury level is likely to decrease the outcome $k$.

Two parameters are used to evaluate the goodness-of-fit of the MNL, Akaike's information criterion (AIC), and likelihood ratio. The model with the smaller AIC is considered the better-fitting model. The confidence level is $95 \%$ for the explanatory variables.

\section{Data Collection}

The six-year crash data (2005 to 2010) were obtained from Florida Crash Annual Report (CAR) System. The CAR system contains a variety of information including driver conditions, vehicle conditions, roadway environmental conditions 
and crash conditions. After cleaning these data with missing codes, a total of 45,798 crashes were occurred at the freeways.

Twenty-eight variables were initially selected and described in Table 1, included one categorical variable, twenty-one dummy variables and five continuous variables. Five injury level outcomes were coded from 1 to 5 . The roadway conditions include the area types (rural/urban), the road surface condition (dry/wet), the type of road (divided/not divided), the road condition (defect/no defect), the road shoulder width (ft), AADT (average annual daily traffic) in thousand, the percentage of heavy truck, maximal post speed limit, lighting conditions (light/no light), and weather conditions (rain/fog).

Crash conditions include the crash types defined by the first harmful events (rear-end, head-on, angle, left-turn, right-turn, sideswipe, and collisions with fixed objects), vehicle type (single vehicle or multi-vehicles), safety equipment used when crash occurred, and vision blockage when crash occurred. Driver information includes age, alcohol and/or drug involved when crash occurred. Vehicle information contains the type of vehicle involved in the crash (auto/van, truck/bus, or bike).

\section{Data Analysis}

1) Multinomial Model Result

Table 2 lists the final model results with the explanatory variables at a 5\% significant level. Five injury outcomes were used and the baseline category is no injury. The variable, fog, is not significant. It is possible that the sample sizes of crashes during fog was relatively small, ranging from $0.5 \%$ to $1.3 \%$ of the total crashes. The road defect is also not significant for the injury outcomes which indicate the variable associated with the number of the crashes but not contributed to injury outcomes. Automobile and truck are found to be less likely involved in four injury levels.

As listed in Table 2, twenty-three variables out of twenty-eight are significant for most of the injury outcomes. Area type is only significant for the incapacitating and fatal injury outcomes. Crashes occur at rural freeways areas are likely to be involved in severe injury levels. Similar to post speed limit, the higher speed limit, the likelihood of severe crashes could occur. AADT is only significant for the possible injury and fatal injury.

One important finding is that increasing the percentage of heavy truck significantly increases the possibility of night fatal crashes. Drivers under drugs or alcohol influenced are more likely to involve in injury crashes except the possible injury. The negative signs for the variable lighting indicate that installing street light could significantly reduce the incapacitating and fatal injury during night time. Vision blocked only significant for possible and non-incapacitating injury and single vehicle crashes are likely for drivers to be involved in higher injury levels at freeway segment. Drivers involving in rear-end, head-on, angle, and left-turn crashes are likely to have severe injuries. 
Table 1. Selected explanatory variables.

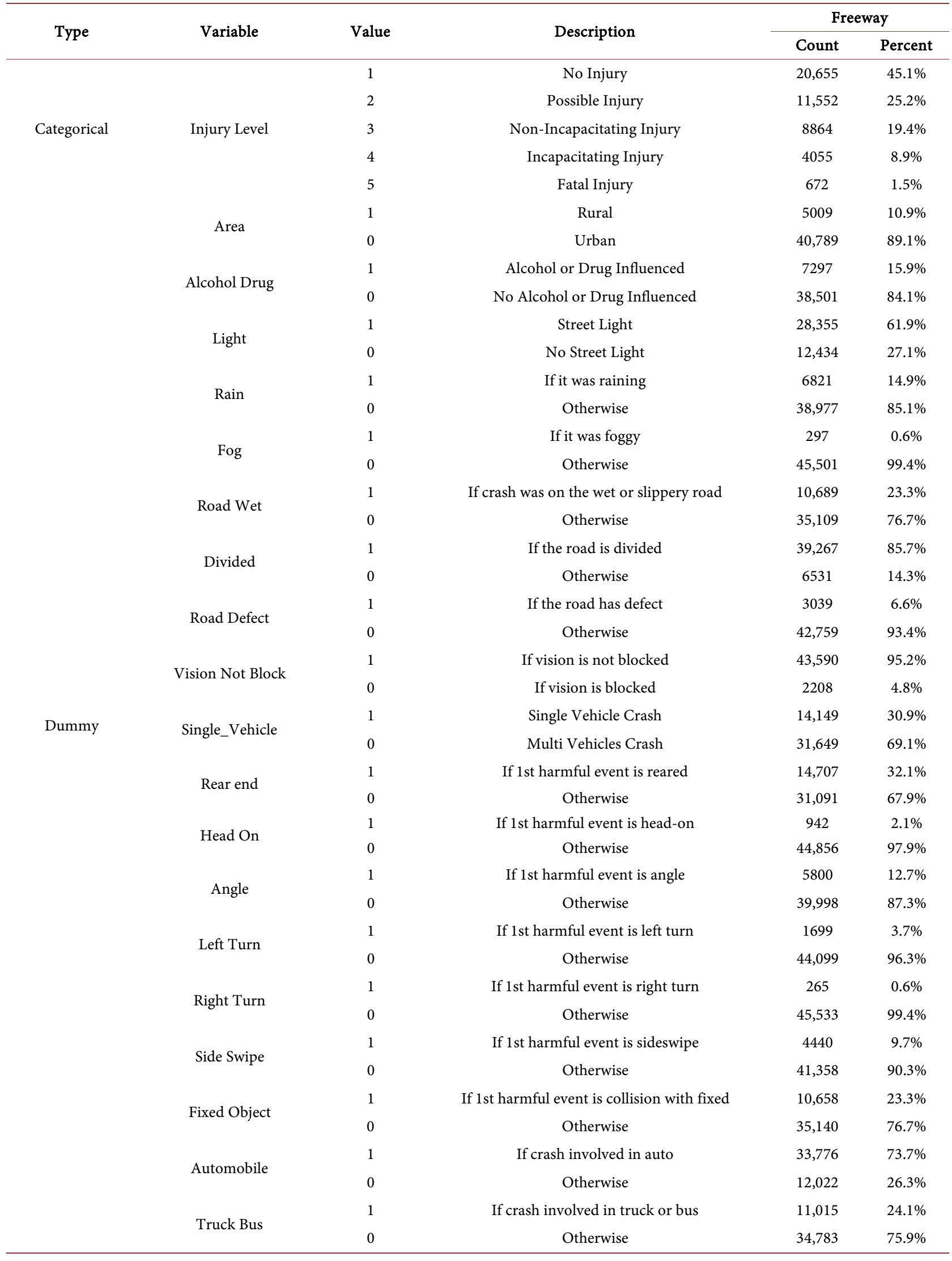




\begin{tabular}{|c|c|c|c|c|c|}
\hline & \multirow{2}{*}{ Bike } & 1 & If crash involved in bike & 130 & $0.3 \%$ \\
\hline & & 0 & Otherwise & 45,668 & $99.7 \%$ \\
\hline & \multirow{2}{*}{ No Safety Protection } & 1 & If safety equipment is not used & 3957 & $8.6 \%$ \\
\hline & & 0 & Otherwise & 41,841 & $91.4 \%$ \\
\hline \multirow{5}{*}{ Continuous } & Should Width & & Road Shoulder Width (ft) & \multicolumn{2}{|c|}{$0-98$} \\
\hline & AADT & & Average Annual Daily Traffic in Thousand & \multicolumn{2}{|c|}{$4.50-328$} \\
\hline & Truck Factor & & Percent of heavy truck (\%) & \multicolumn{2}{|c|}{$0-53$} \\
\hline & Post Speed & & Posted speed limit (mph) & \multicolumn{2}{|c|}{$45-70$} \\
\hline & Age & & Driver age (year) & \multicolumn{2}{|c|}{$15-109$} \\
\hline
\end{tabular}

Table 2. Multinomial Logit model result.

\begin{tabular}{|c|c|c|c|c|c|c|c|c|c|c|c|c|}
\hline \multicolumn{13}{|c|}{ Model Fit Statistics } \\
\hline Observations & \multicolumn{3}{|c|}{45,798} & \multicolumn{3}{|c|}{ Test } & \multicolumn{3}{|c|}{ Chi-Square } & \multicolumn{3}{|c|}{$\operatorname{Pr}>x^{2}$} \\
\hline$A I C$ & \multicolumn{3}{|c|}{113780.3} & \multicolumn{3}{|c|}{ Likelihood Ratio } & \multicolumn{3}{|c|}{5585.886} & \multicolumn{3}{|c|}{$<0.0001$} \\
\hline \multicolumn{13}{|c|}{ Model Analysis } \\
\hline \multirow{2}{*}{ Parameter } & \multicolumn{3}{|c|}{ Possible Injury } & \multicolumn{3}{|c|}{ Non-Incapacitating Injury } & \multicolumn{3}{|c|}{ Incapacitating Injury } & \multicolumn{3}{|c|}{ Fatal Injury } \\
\hline & Coef. & Std. Error & $\operatorname{Pr}>\chi^{2}$ & Coef. & Std. Error & $\operatorname{Pr}>\chi^{2}$ & Coef. & Std. Error & $\operatorname{Pr}>\chi^{2}$ & Coef. & Std. Error & $\operatorname{Pr}>x^{2}$ \\
\hline Intercept & 0.00 & 0.19 & 0.98 & 0.83 & 0.18 & $<0.0001$ & -0.69 & 0.22 & 0.00 & -4.42 & 0.50 & $<0.0001$ \\
\hline Area & - & - & - & - & - & - & 0.27 & 0.06 & $<0.0001$ & 0.21 & 0.13 & 0.11 \\
\hline Should Width & - & - & - & - & - & - & 0.03 & 0.01 & 0.00 & 0.09 & 0.02 & $<0.0001$ \\
\hline $\mathrm{AADT}$ & 0.002 & 0.000 & $<0.0001$ & - & - & - & - & - & - & 0.002 & 0.001 & 0.024 \\
\hline Truck Factor & - & - & - & - & - & - & - & - & - & 0.02 & 0.01 & 0.03 \\
\hline Post Speed & - & - & - & 0.01 & 0.00 & $<0.0001$ & 0.03 & 0.00 & $<0.0001$ & 0.03 & 0.01 & $<0.0001$ \\
\hline Alcohol Drug & -0.17 & 0.03 & $<0.0001$ & 0.11 & 0.04 & 0.00 & 0.11 & 0.05 & 0.02 & 1.33 & 0.09 & $<0.0001$ \\
\hline Lighting & - & - & - & - & - & - & -0.15 & 0.05 & 0.00 & -0.39 & 0.11 & 0.00 \\
\hline Rain & - & - & - & -0.28 & 0.06 & $<0.0001$ & -0.19 & 0.08 & 0.02 & - & - & - \\
\hline Road Wet & 0.09 & 0.04 & 0.02 & - & - & - & -0.18 & 0.07 & 0.01 & - & - & - \\
\hline Divided & 0.08 & 0.04 & 0.02 & 0.11 & 0.04 & 0.01 & - & - & - & 0.82 & 0.19 & $<0.0001$ \\
\hline Vision Block & -0.12 & 0.06 & 0.04 & -0.22 & 0.07 & 0.00 & - & - & - & - & - & - \\
\hline Single Vehicle & 0.40 & 0.07 & $<0.0001$ & 0.61 & 0.07 & $<0.0001$ & 0.60 & 0.08 & $<0.0001$ & 0.37 & 0.16 & 0.02 \\
\hline Rear End & 0.66 & 0.05 & $<0.0001$ & 0.24 & 0.05 & $<0.0001$ & - & - & - & - & - & - \\
\hline Head On & 0.72 & 0.09 & $<0.0001$ & 0.77 & 0.10 & $<0.0001$ & 0.95 & 0.13 & $<0.0001$ & 1.17 & 0.25 & $<0.0001$ \\
\hline Angle & 0.51 & 0.06 & $<0.0001$ & 0.63 & 0.06 & $<0.0001$ & 0.57 & 0.08 & $<0.0001$ & - & - & - \\
\hline Left Turn & 0.79 & 0.08 & $<0.0001$ & 0.96 & 0.08 & $<0.0001$ & 1.13 & 0.11 & $<0.0001$ & - & - & - \\
\hline Right Turn & - & - & - & -0.48 & 0.21 & 0.02 & -0.83 & 0.37 & 0.03 & - & - & - \\
\hline SideSwipe & -0.28 & 0.06 & $<0.0001$ & -0.59 & 0.07 & $<0.0001$ & -0.89 & 0.10 & $<0.0001$ & -2.01 & 0.27 & $<0.0001$ \\
\hline Fixed Object & -0.23 & 0.06 & $<0.0001$ & -0.41 & 0.05 & $<0.0001$ & -0.47 & 0.06 & $<0.0001$ & -0.63 & 0.13 & $<0.0001$ \\
\hline Automible & -1.05 & 0.16 & $<0.0001$ & -2.46 & 0.13 & $<0.0001$ & -3.13 & 0.14 & $<0.0001$ & -3.57 & 0.19 & $<0.0001$ \\
\hline Truck Bus & -1.23 & 0.16 & $<0.0001$ & -2.64 & 0.14 & $<0.0001$ & -3.17 & 0.14 & $<0.0001$ & -3.42 & 0.20 & $<0.0001$ \\
\hline Bike & 1.34 & 0.43 & 0.00 & - & - & - & - & - & - & - & - & - \\
\hline No Safety Protection & -0.13 & 0.05 & 0.01 & 0.40 & 0.05 & $<0.0001$ & 1.04 & 0.05 & $<0.0001$ & 2.09 & 0.09 & $<0.0001$ \\
\hline Age & -0.003 & 0.001 & $<0.0001$ & -0.004 & 0.001 & $<0.0001$ & -0.003 & 0.001 & 0.034 & - & - & - \\
\hline
\end{tabular}


On the contrary, sideswipe and collisions with fixed objects are less likely for the drivers of being involved in the injury outcomes. For the vehicle type, crashes with bikes involved are found to be significant likely to involve in possible injury. It is intuitive that not using safety equipment (seat belt, air bag, helmet, etc.) during the crash significantly increases the possibility of non-incapacitating injury, incapacitating injury, and fatal injury. The finding suggests that the using these safety protections is extremely important to reduce the injury levels, especially the fatal injury at night.

The result for the variable, age, is counter-intuitive. Younger drivers tend to be more likely involved in non-fatal injuries. This finding is quite different from the previous study [5]. It might be reason that the high percentages of young drivers are alcohol or drug involved during the night time. During the night time, drivers are likely to operate at a relatively high speed, younger drivers are easily get distracted and have less driving experience compared to senior drivers. Thus, a more severe consequence might occur at night.

The signs for the four variables, shoulder width, rain, wet, and divided, are a little counter intuitive. For shoulder width on the right side, it could be a variable associated with road type, while the road shoulder is generally wider in the rural area than the urban area where severe injury levels are likely to occur. When it was raining or the road condition is wet, drivers are less likely to involve in incapacitating injury. The probable reason could be the driver behavior when drivers might be more cautious if it was raining or the road was wet. It might also correlate with the traveling speed, drivers usually travel at a relatively lower speed during these conditions which are associated with less severe injury outcomes.

The positive sign for the divided indicate the divided construction in the median are likely for drivers of being possible injury, non-incapacitating injury and fatal injury compared to when the median is open to grass. It is possibility that drivers could operate or take evasive maneuvers during a crash in a median without barrel or divided construction.

2) Analysis of Odds Ratios

The odds ratios were computed for each significant explanatory variable, as listed in Table 3. The ratios represent the probability of one injury outcome over the probability of the base injury outcome (no injury) for increasing one unit of an explanatory variable while others hold constant. If OR is greater than 1 , it is likely increasing the probabilities of injury outcomes; if OR is less than 1 , it is likely to decrease the probabilities of the injury outcomes. For example, the probability of being in a fatal injury from urban to rural area is 1.23 times as no injury for freeways. The odds ratios for posted speed limit are larger than one which indicates the higher post speed limits slightly increase the injury outcomes at the freeway segments.

AADT has a slight impact on the injury outcomes only at freeway. One thousand vehicles increasing result in 1.002 times the probability of fatal injury at freeway as no injury outcome. Drivers under alcohol or drug influenced are 
Table 3. Calculated odds ratio for injury levels vs. no injury.

\begin{tabular}{|c|c|c|}
\hline Parameter & Injury Levels & Ratio \\
\hline \multirow{4}{*}{ Shoulder Width } & Incapacitating Injury & 1.307 \\
\hline & Fatal Injury & 1.230 \\
\hline & Incapacitating Injury & 1.026 \\
\hline & Fatal Injury & 1.090 \\
\hline \multirow{6}{*}{$\mathrm{AADT}$} & Possible Injury & 1.002 \\
\hline & Fatal Injury & 1.002 \\
\hline & Fatal Injury & 1.020 \\
\hline & Non Incapacitating Injury & 1.013 \\
\hline & Incapacitating Injury & 1.029 \\
\hline & Fatal Injury & 1.032 \\
\hline \multirow{8}{*}{ Alcohol Drug } & Possible Injury & 0.845 \\
\hline & Non Incapacitating Injury & 1.113 \\
\hline & Incapacitating Injury & 1.114 \\
\hline & Fatal Injury & 3.770 \\
\hline & Incapacitating Injury & 0.864 \\
\hline & Fatal Injury & 0.676 \\
\hline & Non Incapacitating Injury & 0.753 \\
\hline & Incapacitating Injury & 0.823 \\
\hline \multirow{2}{*}{ Road Wet } & Possible Injury & 1.099 \\
\hline & Incapacitating Injury & 0.832 \\
\hline \multirow{3}{*}{ Divided } & Possible Injury & 1.088 \\
\hline & Non Incapacitating Injury & 1.117 \\
\hline & Fatal Injury & 2.281 \\
\hline \multirow{3}{*}{ Vision Block } & Possible Injury & 0.885 \\
\hline & Non Incapacitating Injury & 0.804 \\
\hline & Possible Injury & 1.489 \\
\hline \multirow{3}{*}{ Single Vehicle } & Non Incapacitating Injury & 1.839 \\
\hline & Incapacitating Injury & 1.821 \\
\hline & Fatal Injury & 1.441 \\
\hline \multirow{2}{*}{ Rear end } & Possible Injury & 1.931 \\
\hline & Non Incapacitating Injury & 1.277 \\
\hline \multirow{4}{*}{ Head On } & Possible Injury & 2.048 \\
\hline & Non Incapacitating Injury & 2.156 \\
\hline & Incapacitating Injury & 2.595 \\
\hline & Fatal Injury & 3.227 \\
\hline \multirow{3}{*}{ Angle } & Possible Injury & 1.661 \\
\hline & Non Incapacitating Injury & 1.878 \\
\hline & Incapacitating Injury & 1.770 \\
\hline
\end{tabular}




\section{Continued}

\begin{tabular}{|c|c|c|}
\hline \multirow{4}{*}{ Left Turn } & Possible Injury & 2.201 \\
\hline & Non Incapacitating Injury & 2.606 \\
\hline & Incapacitating Injury & 3.105 \\
\hline & Fatal Injury & 1.231 \\
\hline \multirow{4}{*}{ Right Turn } & Possible Injury & 0.893 \\
\hline & Non Incapacitating Injury & 0.622 \\
\hline & Incapacitating Injury & 0.438 \\
\hline & Fatal Injury & $<0.001$ \\
\hline \multirow{4}{*}{ Side Swipe } & Possible Injury & 0.758 \\
\hline & Non Incapacitating Injury & 0.554 \\
\hline & Incapacitating Injury & 0.412 \\
\hline & Fatal Injury & 0.135 \\
\hline \multirow{4}{*}{ Fixed Object } & Possible Injury & 0.795 \\
\hline & Non Incapacitating Injury & 0.667 \\
\hline & Incapacitating Injury & 0.625 \\
\hline & Fatal Injury & 0.531 \\
\hline \multirow{4}{*}{ Automobile } & Possible Injury & 0.349 \\
\hline & Non Incapacitating Injury & 0.086 \\
\hline & Incapacitating Injury & 0.044 \\
\hline & Fatal Injury & 0.028 \\
\hline \multirow{4}{*}{ Truck Bus } & Possible Injury & 0.293 \\
\hline & Non Incapacitating Injury & 0.072 \\
\hline & Incapacitating Injury & 0.042 \\
\hline & Fatal Injury & 0.033 \\
\hline Bike & Possible Injury & 3.811 \\
\hline \multirow{4}{*}{ No Safety Protection } & Possible Injury & 0.879 \\
\hline & Non Incapacitating Injury & 1.493 \\
\hline & Incapacitating Injury & 2.832 \\
\hline & Fatal Injury & 8.118 \\
\hline \multirow{3}{*}{ Age } & Possible Injury & 0.997 \\
\hline & Non Incapacitating Injury & 0.996 \\
\hline & Incapacitating Injury & 0.997 \\
\hline
\end{tabular}

found more likely to be killed, up to 3.77 times at freeways compared to no alcohol or drug influenced. The ORs for lighting are smaller than 1.0. The results suggest the having street lights could reduce the relative risk of being in incapacitating injury and fatal injury. The probabilities decreased to 0.7 times for the probability of being killed at freeways as the probability of no injury. 


\section{Conclusions}

This study investigated the crash contributing factors to the injury outcomes and the characteristics of the night time crashes at freeways mainline sections. The multinomial logit model was developed to estimate the contributing factors, including driver conditions, geometric conditions, vehicle conditions, crash conditions, and environmental conditions, to different injury outcomes for night time crashes. The six-year crash data (2005-2010) were obtained from Florida Crash Annual Report (CAR) System. The data were divided into five datasets based on the five identified locations. Five injury level outcomes, no injury, possible injury, non-incapacitating injury, capacitating injury, and fatal injury, were considered. The no injury was selected as the baseline category. A total of 45,798 crashes were observed at freeways.

Nighttime crashes at rural areas are likely to be involved in incapacitating injury and fatal injury. AADT is only significant for the possible injury and fatal injury. Vision blocked only significant for possible and non-incapacitating injury. Drivers involving in rear-end, head-on, angle, and left-turn crashes are likely to suffer in severe injuries. Younger drivers tend to be involved in more non-fatal injuries. It might be contributed by many young drivers during the night time.

When it was raining or the road condition was wet, drivers are less likely to involve in incapacitating injury. The presence of the divided constructions in the median is likely for drivers of being possible injury, non-incapacitating injury and fatal injury compared to when the median is open to grass. For shoulder width on the right side, it could be a variable associated with road type and driver behavior, while the road shoulder is generally wider in the rural area than the urban area where high injury level is likely to occur.

The probability of being in a fatal injury from urban to rural area is 1.23 times as no injury for freeways. Drivers under alcohol or drug influenced are found more likely to be killed, up to 3.77 times at freeways. The probabilities of having street lights decreased to 0.7 times for fatal crashes at freeways to the probability of no injury. Head-on, angle, left-turn crashes are relatively high risky crash types, ranging from 2 to 18 times of the probability of severe injury outcomes (non-incapacitating injury, incapacitating injury, and fatal injury) while right-turn, and sideswipe have lower risks ranging from 0.001 to 0.9 times to the no injury.

Having safety protection equipment is a key factor to reduce the injury outcomes. For fatal injury only, it increases the probability up to 8 times at freeway, when no safety equipment was used compared to the protection equipment used. Drivers involving in single vehicle crashes are more likely to be killed which is 1.4 times at freeway segment. Younger people are more likely to increase the probability of being non-fatal injury at freeways.

One interesting finding is divided that highways are more likely to involve in fatal crashes. Drivers being killed are 2.28 times at freeways than being involved in no injury crashes. This variable might correlate with other factors, like higher 
operating speeds, less cautious and more distracted at divided highways compared to undivided ones.

The method can be applied to other types of road conditions, ramps, intersections, major and minor arterials, etc. The findings from this study could help the engineers and researchers to further select the effective countermeasures or policies to potentially reduce the night time crashes, especially the injury severity levels.

The authors suggest more efforts should be made to increase the using of safety equipment, e.g. safety belt, air bag, helmet, at night which could significantly reduce the possibilities of injury outcomes, especially fatal injury levels. Efforts on awareness of risks of speeding, alcohol, or drug impacts, is also strongly recommended. Installation of street light is the most effective way to reduce the injury outcomes. Young drivers should be aware of the risk of driving at freeway. Further investigations on these divided highways are strongly recommended to find the possible reasons of high probability of injury outcomes compared to not divided highways.

\section{Conflicts of Interest}

The authors declare no conflicts of interest regarding the publication of this paper.

\section{References}

[1] National Highway Traffic Safety Association (NHTSA) (2007) Passenger Vehicle Occupant Fatalities by Day and Night-A Contrast. Washington DC.

[2] Hawkins, N., Hallmark, S., Smadi, O., Kinsenbaw, C., Orellana, M., Hans, Z. and Isebrands, H. (2008) Strategies to Address Nighttime Crashes at Rural, Unsignalized Intersections. Final Report, Center for Transportation Research and Education, Iowa State University, Ames.

[3] Elvik, R. (1997) Meta-Analysis of Evaluations of Public Lighting as Accident Countermeasure. Transportation Research Record, 1485, 112-123.

[4] Griffith, S.M. (1994) Comparison of the Safety of Lighting Options on Urban Freeways. Public Roads, 58, 8-15.

[5] Ye, F. and Lord, D. (2014) Comparing Three Commonly Used Crash Severity Models on Sample Size Requirements: Multinomial Logit, Ordred Probit and Mixed Logit Models. Analytic Methods in Accident Research, 1, 72-85.

[6] Xie, Y., Zhao, K. and Huynh, N. (2012) Analysis of Driver Injury Severity in Rural Single-Vehicle Crashes. Accident Analysis and Prevention, 47, 36-44.

https://doi.org/10.1016/j.aap.2011.12.012

[7] Geedipally, S.R., Turner, P.A. and Sunil, P. (2011) Analysis of Motorcycle Crashes in Texas with Multinomial Logit Model. Transportation Research Record, 2265, 62-69. https://doi.org/10.3141/2265-07

[8] Bham, H.G., Javvadi, S.B. and Manepalli, R.R.U. (2012) Multinomial Logistic Regression Model for Single-Vehicle and Multivehicle Collisions on Urban U.S. Highways in Arkansas. Journal of Transportation Engineering, American Society of Civil Engineers, 138, 786-797. https://doi.org/10.1061/(ASCE)TE.1943-5436.0000370

[9] Kim, J.-K., Ulfarsson, G.V. and Kim, S. (2008) Age and Pedestrian Injury Severity in 
Motor-Vehicle Crashes: A Heteroskedastic Logit Analysis. Accident Analysis and Prevention, 40, 1695-1702. https://doi.org/10.1016/j.aap.2008.06.005

[10] Gumbel, E. (1958) Statistics of Extremes. Columbia University Press, New York. https://doi.org/10.7312/gumb92958 